\title{
A METHOD FOR IMAGING CORROSION DAMAGE IN THIN PLATES FROM ELECTROSTATIC DATA
}

\author{
Peter G. KauP ${ }^{1}$ \\ Department of Mathematical Sciences \\ University of Delaware \\ Newark, DE 19716 \\ FADIL SANTOSA ${ }^{2}$ \\ Advanced Computing Research Institute \\ Cornell Theory Center \\ Cornell University \\ Ithaca, NY 14853 \\ Michael Vogelius ${ }^{3}$ \\ Department of Mathematics \\ Rutgers University \\ New Brunswick, NJ 08943
}

\begin{abstract}
The problem of quantitative nondestructive evaluation of corrosion in plates is considered. The inpection method uses boundary measurements of currents and voltages to determine the material loss caused by corrosion. The development of the method is based on linearization and the assumption that the plate is thin. The behavior of the method is examined in numerical simulations.
\end{abstract}

Key words. nondestructive evaluation, corrosion monitoring, electrical impedance tomography, numerical reconstruction methods

\footnotetext{
${ }^{1}$ Research partially supported by an NSF Mathematical Sciences Graduate Fellowship and by the Department of Energy under grant DE-FG02-94ER25225.

2 Research partially supported by the Air Force Office of Scientific Research under a URI-RIP grant F4962093-I-0500, the Department of Energy under grant DE-FG02-94ER25225, the National Science Foundation under grant DMS-9210489, and in part by the Cornell Theory Center which receives major funding from the National Science Foundation and IBM corporation, with additional support from New York State and members of its Corporate Research Institute. Permanent address: Department of Mathematical Sciences and Department of Mechanical Engineering, University of Delaware, Newark, DE 19716.

${ }^{3}$ Research partially supported by the National Science Foundation under grant NSF DMS-9202042, the Air Force Office of Scientific Research under grants 89NM605 and under a URI-RIP grant F49620-93-I-0500.
} 
1. Introduction. The purpose of this work is to demonstrate that a nondestructive evaluation technique based on electrical impedance tomography can be effectively applied to image corrosion damage in thin plates. Electrical impedance tomography uses static voltage and current measurements on the surface of a specimen to determine the conductivity distribution in the interior. In this paper, we develop a method to determine material loss occuring on the inaccessible side of a thin plate by measuring voltages and currents on the opposite (accessible) side. The method leads to quantitative reconstruction of the loss profile.

In some ways, the present method is similar to the classical potential drop method [5] which is frequently used to determine the depth of a surface crack. One major difference is that the collected data are used to reconstruct the loss profile on the side opposite the measurements.

The method requires highly accurate voltage readings: 4 to 5 significant figures. In turn, this seems sufficient to reconstruct a material loss of around 5 per cent. While such a level of measurement accuracy may appear difficult to achieve in general, we believe that due to the simplicity of the present procedure, data of this quality is realistic.

We model damage by corrosion as material loss, leading to an unknown profile of the inaccessible side of the plate. This model is somewhat simplistic but serves as a good starting point for further research. A two-dimensional version of this problem was studied in Kaup and Santosa [7]. We also note that the question of identifiability in two dimensions has been addressed in the work of Andrieux et al [2].

In the present study, the corroded surface is considered perfectly insulated. A more realistic boundary condition, which takes into account the chemical reduction and oxidation which takes place, is the focus of [8]. Our reconstruction algorithm is based on the linearization of the relationship between the voltage data and the function describing the loss profile. Linearization is justified when material loss is small in relation to the plate thickness - for instance, of the order of 5 $10 \%$. The assumption of a thin plate geometry affords additional simplification. The simplified relationship between the loss profile and the measured data forms the basis for the reconstruction scheme. We show that by careful regularization, a simple and effective algorithm can be devised. The performance of the algorithm is demonstrated in several numerical examples.

2. Model of problem. For simplicity, we consider a (flat) plate geometry. Much of what we do in this paper can be extended to more general geometries (for instance, shells). As mentioned in the introduction, we model corrosion as material loss. We are well aware that real corrosion is much more complicated [1].

Consider an undamaged plate, $\Omega_{0}$, given by

$$
\Omega_{0}=\left\{\left(x_{1}, x_{2}, x_{3}\right):\left|x_{1}\right| \leq 1 / 2,\left|x_{2}\right| \leq 1 / 2,0 \leq x_{3} \leq a\right\} .
$$

Corrosion has occured on the upper, inaccessible, surface $x_{3}=a$. We assume that it has caused the plane $x_{3}=a$ to become $x_{3}=a+\epsilon \theta\left(x_{1}, x_{2}\right)$. The damaged plate is therefore given by

$$
\Omega_{\epsilon}=\left\{\left(x_{1}, x_{2}, x_{3}\right):\left|x_{1}\right| \leq 1 / 2,\left|x_{2}\right| \leq 1 / 2,0 \leq x_{3} \leq a+\epsilon \theta\left(x_{1}, x_{2}\right)\right\} .
$$

The goal is to determine the loss profile $\epsilon \theta\left(x_{1}, x_{2}\right)$. In order to do so, we create a voltage potential by applying a current pattern to the bottom of the plate, $x_{3}=0$. The resulting voltage potential is also measured on the bottom. We seek to determine $\epsilon \theta\left(x_{1}, x_{2}\right)$ from these measurement of the voltage potential $u\left(x_{1}, x_{2}, 0\right)=g\left(x_{1}, x_{2}\right)$.

We will not be specific at this time about the applied current pattern. The analysis of the problem will lead to an obvious and physically reasonable choice of pattern. We shall assume that the measurements are available at a rectangular array of points on the plate bottom. 
The potential $u\left(x_{1}, x_{2}, x_{3}\right)$ satisfies Laplace's equation in the damaged plate

$$
\triangle u=0 \text { in } \Omega_{\epsilon} .
$$

The boundary conditions, aside from the applied current pattern on the bottom of the plate, are homogeneous Neumann boundary conditions corresponding to perfectly insulated boundaries. Therefore

$$
\begin{aligned}
& \frac{\partial u}{\partial x_{i}}=0 \quad \text { on } \quad x_{i}= \pm 1 / 2, \quad i=1,2 \\
& \frac{\partial u}{\partial \nu}=0 \quad \text { on } \quad x_{3}=a+\epsilon \theta\left(x_{1}, x_{2}\right),
\end{aligned}
$$

and

$$
\frac{\partial u}{\partial x_{3}}=-\phi\left(x_{1}, x_{2}\right) \quad \text { on } \quad x_{3}=0 .
$$

Here $\nu$ is the outward normal to the surface $x_{3}=a+\epsilon \theta\left(x_{1}, x_{2}\right)$. The solution to the boundary value problem (1) is unique up to a constant. To make the solution unique, we choose the normalization $\int_{-\frac{1}{2}}^{\frac{1}{2}} \int_{-\frac{1}{2}}^{\frac{1}{2}} u\left(x_{1}, x_{2}, 0\right) d x_{1} d x_{2}=0$.

The choice of the input current is at our disposal, we will have more to say about this choice later. We could make several sets of measurements corresponding to different input currents, but it seems likely that one set of measurements should suffice to determine $\epsilon \theta\left(x_{1}, x_{2}\right)$.

3. Linearization. Let $\omega=\left\{\left(x_{1}, x_{2}\right)\right.$ : $\left.\left|x_{1}\right| \leq 1 / 2,\left|x_{2}\right| \leq 1 / 2\right\}$. Using Green's first identity, we can rewrite the boundary value problem (1) in variational form as

$$
\begin{aligned}
\int_{\Omega_{\epsilon}}\left(\nabla_{x} u \cdot \nabla_{x} v\right) d x & =\int_{\partial \Omega_{\epsilon}}\left(\frac{\partial u}{\partial n} v\right) d \sigma_{x} \\
& =\int_{\omega} \phi\left(x_{1}, x_{2}\right) v\left(x_{1}, x_{2}, 0\right) d x_{1} d x_{2}
\end{aligned}
$$

where $v\left(x_{1}, x_{2}, x_{3}\right) \in H^{1}\left(\Omega_{\epsilon}\right)$ is an arbitrary test function.

The variational equation, however, requires integration over the unknown region. Therefore, we use a change of coordinates $z=\Phi_{\epsilon}(x)$ given by

$$
z_{1}=x_{1}, \quad z_{2}=x_{2}, \quad z_{3}=x_{3}-\epsilon \theta\left(x_{1}, x_{2}\right) \gamma\left(x_{3}\right),
$$

where $\gamma(t)$ is a smooth function which satisfies $\gamma(t)=0$ for $t$ near 0 , and $\gamma(t)=1$ for $t$ near $a$. For $\epsilon$ sufficiently small this coordinate transformation maps the domain $\Omega_{\epsilon}$ to the reference (undamaged) domain $\Omega_{0}$. See Figure 1 .

The Jacobian matrix for this transformation is

$$
\left[\frac{d \Phi_{\epsilon}}{d x}\right]=I-\epsilon \delta J
$$

with the matrix $\delta J$ given by

$$
\delta J=\left[\begin{array}{ccc}
0 & 0 & 0 \\
0 & 0 & 0 \\
\frac{\partial \theta}{\partial x_{1}} \gamma & \frac{\partial \theta}{\partial x_{2}} \gamma & \theta \dot{\gamma}
\end{array}\right]
$$



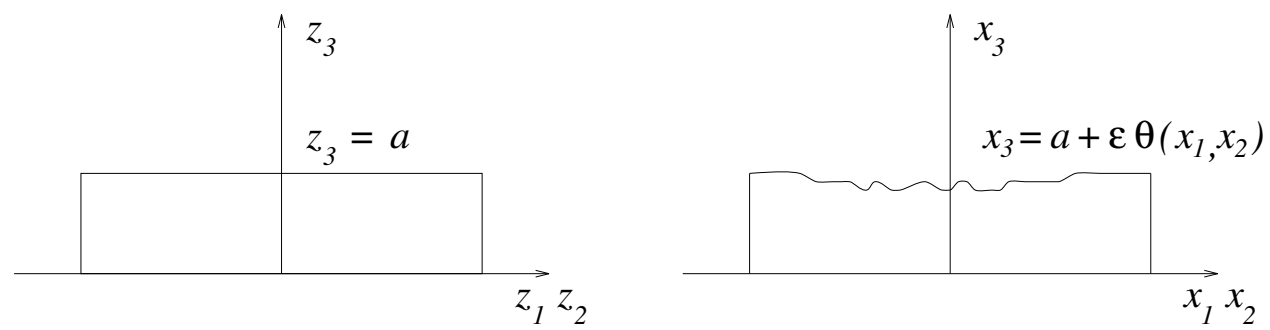

FIG. 1. The coordinate change $x \rightarrow z$ maps the damaged plate $\Omega_{\epsilon}$ to the undamaged plate $\Omega_{0}$.

The corresponding determinant is

$$
\operatorname{det}\left[\frac{d \Phi_{\epsilon}}{d x}\right]=1-\epsilon \theta \dot{\gamma}
$$

For $\epsilon \ll a<1$ the map $\Phi_{\epsilon}: x \rightarrow z$ thus represents a regular change of variables, and we also have

$$
\operatorname{det}\left[\frac{d \Phi_{\epsilon}^{-1}}{d z}\right]=(1+\epsilon \theta \dot{\gamma}) \circ \Phi_{\epsilon}^{-1}+O\left(\epsilon^{2}\right) .
$$

Applying this change of variables to (2), we now obtain:

$$
\begin{aligned}
\int_{\omega} \phi\left(z_{1}, z_{2}\right) v\left(z_{1}, z_{2}, 0\right) d z_{1} d z_{2} & =\int_{\Omega_{0}}\left\{\left(\nabla_{z} u \cdot \nabla_{z} v\right)\right. \\
-\epsilon \nabla_{z} u & \left.\cdot\left(\delta J+\delta J^{T}\right) \circ \Phi_{\epsilon}^{-1} \nabla_{z} v\right\}(1+\epsilon \theta \dot{\gamma}) \circ \Phi_{\epsilon}^{-1} d z+O\left(\epsilon^{2}\right) .
\end{aligned}
$$

For small $\epsilon$, we formally expand the voltage potential $u(z)$ as:

$$
u=u_{0}+\epsilon u_{1}+O\left(\epsilon^{2}\right) .
$$

The term $u_{0}$ is the solution to the boundary value problem for the undamaged reference body. Similarly, the term $\epsilon u_{1} \approx u-u_{0}$ is the $O(\epsilon)$ voltage change resulting from the surface perturbation $\epsilon \theta$.

Substituting this expansion back into equation (3), we obtain

$$
\begin{aligned}
& \int_{\omega} \phi\left(z_{1}, z_{2}\right) v\left(z_{1}, z_{2}, 0\right) d z_{1} d z_{2}=\int_{\Omega_{0}} \nabla_{z} u_{0} \cdot \nabla_{z} v d z \\
& +\epsilon \int_{\Omega_{0}}\left\{\begin{array}{c}
\nabla_{z} u_{1} \cdot \nabla_{z} v+\nabla_{z} u_{0} \cdot \nabla_{z} v \theta \dot{\gamma} \circ \Phi_{0}^{-1} \\
\left.-\nabla_{z} u_{0} \cdot\left(\delta J+\delta J^{T}\right) \circ \Phi_{0}^{-1} \nabla_{z} v\right\} d z+O\left(\epsilon^{2}\right) .
\end{array}\right.
\end{aligned}
$$

The $O(1)$ terms in this identity balance since $u_{0}$ is the solution to (1) for $\epsilon=0$. Examining the $O(\epsilon)$ terms (using the fact that $\Phi_{0}^{-1}=I$ ), we find that $u_{1}$ must satisfy the equation

$$
\begin{aligned}
& \int_{\Omega_{0}} \nabla_{z} u_{1} \cdot \nabla_{z} v d z= \\
& \quad \int_{\Omega_{0}}\left\{\nabla_{z} u_{0} \cdot\left(\delta J+\delta J^{T}\right) \nabla_{z} v-\left(\nabla_{z} u_{0} \cdot \nabla_{z} v\right) \theta \dot{\gamma}\right\} d z
\end{aligned}
$$


The next step involves manipulating (5) so that we end up with an identity between boundary integrals.

After substitution of the exact form of $\delta J$ into (5) and collection of terms, the identity becomes

$$
\begin{aligned}
\int_{\Omega_{0}} \nabla_{z} u_{1} \cdot \nabla_{z} v d z= & \int_{\Omega_{0}} \theta \dot{\gamma}\left[\frac{\partial u_{0}}{\partial z_{3}} \frac{\partial v}{\partial z_{3}}-\frac{\partial u_{0}}{\partial z_{1}} \frac{\partial v}{\partial z_{1}}-\frac{\partial u_{0}}{\partial z_{2}} \frac{\partial v}{\partial z_{2}}\right] d z \\
& +\int_{\Omega_{0}} \gamma \frac{\partial \theta}{\partial z_{1}}\left(\frac{\partial v}{\partial z_{1}} \frac{\partial u_{0}}{\partial z_{3}}+\frac{\partial v}{\partial z_{3}} \frac{\partial u_{0}}{\partial z_{1}}\right) d z \\
& +\int_{\Omega_{0}} \gamma \frac{\partial \theta}{\partial z_{2}}\left(\frac{\partial v}{\partial z_{2}} \frac{\partial u_{0}}{\partial z_{3}}+\frac{\partial v}{\partial z_{3}} \frac{\partial u_{0}}{\partial z_{2}}\right) d z .
\end{aligned}
$$

We now choose $v\left(z_{1}, z_{2}, z_{3}\right)$ to be harmonic in $\Omega_{0}$ and to satisfy the boundary conditions

$$
\begin{gathered}
\frac{\partial v}{\partial z_{i}}=0 \quad \text { on } \quad z_{i}= \pm 1 / 2 \quad i=1,2 \\
\frac{\partial v}{\partial z_{3}}=0 \quad \text { on } \quad z_{3}=a .
\end{gathered}
$$

After integration by parts of both sides of the equation and some manipulations, we arrive at

$$
\int_{\omega} u_{1}\left(z_{1}, z_{2}, 0\right) \frac{\partial v}{\partial z_{3}}\left(z_{1}, z_{2}, 0\right) d z_{1} d z_{2}=\int_{\omega} \theta\left(z_{1}, z_{2}\right)\left(\frac{\partial u_{0}}{\partial z_{1}} \frac{\partial v}{\partial z_{1}}+\frac{\partial u_{0}}{\partial z_{2}} \frac{\partial v}{\partial z_{2}}\right)\left(z_{1}, z_{2}, a\right) d z_{1} d z_{2} .
$$

We shall use this equation as the basis for our solution of the linearized inverse problem. In this equation, $v$ is an arbitrary harmonic function satisfying the boundary conditions (7). The left side represents averaged data whereas the right side represents an operator acting on the unknown $\theta$.

It is worth noting that the choice of both $u_{0}$ and $v$ is at our disposal. The background field $u_{0}$ is determined by the applied current $\phi\left(x_{1}, x_{2}\right)$ and the undamaged domain $\Omega_{0}$. We will choose $\phi$ and $v$ with a view towards obtaining the simplest formula.

As we proceed with the development of our algorithm, we shall change the notation for the integration variable in (8). Henceforth, we shall use $x$ instead of $z$.

4. Thin Plate Approximation. The first-kind integral equation (8) is still somewhat cumbersome to solve numerically. In order to determine for $\theta\left(x_{1}, x_{2}\right)$, we need to have at our disposal a set of harmonic test functions $v\left(x_{1}, x_{2}, x_{3}\right)$. To evaluate the right side of (8), each test function will need to be integrated against the partial derivatives of background field $u_{0}\left(x_{1}, x_{2}, x_{3}\right)$. Each test function gives rise to a single equation. The unknown loss profile is found by solving this set of equations.

To arrive at a much simpler procedure, we consider the regime where $a \ll 1$, that is, the situation where the plate thickness is small. With this approximation, the right side of (8) becomes more explicit. Recall that the linearization is valid for $\epsilon \ll a$. Therefore, the thin plate approximation is justified provided $\epsilon / a \ll 1$ and at the same time $a \ll 1$.

The following calculation will lead to a choice of test functions $v$ and of input current $\phi$ which provides a simplified equation satisfied by $\theta$.

We begin by scaling the variables

$$
\bar{x}=\left(x_{1}, x_{2}\right), \quad x_{3}^{\prime}=x_{3} / a .
$$

This effectively transforms the plate domain $\Omega_{0}=\omega \times[0, a]$ to $\Omega_{0}^{\prime}=\omega \times[0,1]$. Note that

$$
\frac{\partial}{\partial x_{3}}=\frac{1}{a} \frac{\partial}{\partial x_{3}^{\prime}} .
$$


We will use the notation

$$
\bar{\nabla}=\left(\frac{\partial}{\partial x_{1}}, \frac{\partial}{\partial x_{2}}\right), \text { and } \bar{\triangle}=\frac{\partial^{2}}{\partial x_{1}^{2}}+\frac{\partial^{2}}{\partial x_{2}^{2}} .
$$

The requirement that $v$ be harmonic translates to

$$
0=\triangle v=\bar{\triangle} v+a^{-2} \frac{\partial^{2} v}{\partial{x_{3}^{\prime}}^{2}} .
$$

We have the boundary conditions

$$
\frac{\partial v}{\partial x_{i}}=0 \quad \text { on the sides } \quad x_{i}= \pm 1 / 2
$$

on the sides of the plate, and on the top of the plate $\left(x_{3}^{\prime}=1\right)$ we have

$$
\frac{\partial v}{\partial x_{3}}=\frac{1}{a} \frac{\partial v}{\partial x_{3}^{\prime}}=0
$$

On the bottom of the plate $\left(x_{3}^{\prime}=0\right)$ we set

$$
\frac{\partial v}{\partial x_{3}}=a \zeta
$$

which implies that

$$
\frac{\partial v}{\partial x_{3}^{\prime}}(\bar{x}, 0)=a^{2} \zeta(\bar{x})
$$

The function $\zeta(\bar{x})$ is assumed to have compact support inside $\omega$, its exact form will be specified later.

Now, we expand the test functions $v\left(\bar{x}, x_{3}^{\prime}\right)$ in (even) powers of the small parameter $a$

$$
v\left(\bar{x}, x_{3}^{\prime}\right)=v_{0}\left(\bar{x}, x_{3}^{\prime}\right)+a^{2} v_{2}\left(\bar{x}, x_{3}^{\prime}\right)+a^{4} v_{4}\left(\bar{x}, x_{3}^{\prime}\right)+\cdots .
$$

Considering the lowest order terms resulting from the equations (9), we obtain the boundary value problem

$$
\begin{array}{lll}
\frac{\partial^{2} v_{0}}{\partial x_{3}^{\prime 2}}=0 & \text { in } & \Omega_{0}^{\prime} \\
\frac{\partial v_{0}}{\partial \nu}=0 & \text { for } & \bar{x} \in \partial \omega \\
\frac{\partial v_{0}}{\partial x_{3}^{\prime}}=0 & \text { for } & x_{3}^{\prime}=0,1 .
\end{array}
$$

Thus, we see that $v_{0}$ is independent of $x_{3}^{\prime}$ (i.e. $v_{0}=v_{0}(\bar{x})$ ). Considering the next order terms we obtain the system

$$
\begin{aligned}
\bar{\triangle} v_{0}+\frac{\partial^{2}}{\partial x_{3}^{\prime 2} v_{2}}=0 & \text { in } & \Omega_{0}^{\prime} \\
\frac{\partial v_{2}}{\partial \nu}=0 & \text { for } & \bar{x} \in \partial \omega \\
\frac{\partial v_{2}}{\partial x_{3}^{\prime}}=0 & \text { for } & x_{3}^{\prime}=1 \\
\frac{\partial v_{2}}{\partial x_{3}^{\prime}}=\zeta(\bar{x}) & \text { for } & x_{3}^{\prime}=0
\end{aligned}
$$


Since $v_{0}$ is independent of $x_{3}^{\prime}$, we can integrate in $x_{3}^{\prime}$ from 0 to 1 to obtain

$$
\bar{\triangle} v_{0}=\zeta(\bar{x}) \quad \text { for } \quad \bar{x} \in \omega
$$

with

$$
\frac{\partial v_{0}}{\partial \nu}=0 \quad \text { for } \quad \bar{x} \in \partial \omega .
$$

The expansion can be continued to obtain equations for $v_{i}, i=2,4, \ldots$. For our purpose, we stop with the leading order term $v_{0}$, described by (10). We note that the expansion is purely formal. It can be made rigorous with appropriate smoothness assumptions.

By incorporating the identity (9d) into (8), and replacing $v$ by $v_{0}$ on the right side, we obtain

$$
\begin{aligned}
& a \int_{\omega} u_{1}\left(x_{1}, x_{2}, 0\right) \zeta\left(x_{1}, x_{2}\right) d x_{1} d x_{2} \approx \\
& \quad \int_{\omega} \theta\left(x_{1}, x_{2}\right)\left(\frac{\partial u_{0}}{\partial x_{1}}\left(x_{1}, x_{2}, a\right) \frac{\partial v_{0}}{\partial x_{1}}\left(x_{1}, x_{2}\right)+\frac{\partial u_{0}}{\partial x_{2}}\left(x_{1}, x_{2}, a\right) \frac{\partial v_{0}}{\partial x_{2}}\left(x_{1}, x_{2}\right)\right) d x_{1} d x_{2} .
\end{aligned}
$$

The consequences of this approximate identity are studied next. The fact that there is a factor of $a$ on the left side reflects that it might have been more natural to start with a plate in which $\theta$ was proportional to $a$ as well.

5. Implications of the thin plate approximation in 2-D. In our earlier investigation, [7], we observed that when the applied current is concentrated in small regions near the ends of a thin 2-D beam $\left(\omega=\left\{x_{1}:\left|x_{1}\right| \leq 1 / 2\right\}\right)$ then the function $\theta\left(x_{1}\right)$ appears to be proportional to the derivative of $u_{1}\left(x_{1}, 0\right)$. As will be shown, this relationship turns out to be a direct consequence of the approximate equality (11).

We formally choose $\zeta\left(x_{1}\right)=\delta^{\prime}\left(x_{1}-\xi\right)$, the derivative of the Dirac delta function. Integrating the analog of $(10)$, we now obtain

$$
v_{0}^{\prime}\left(x_{1}\right)=\delta\left(x_{1}-\xi\right)
$$

The linearized relation, (11), between $\theta$ and $u_{1}$ reads

$$
a \int_{-\frac{1}{2}}^{\frac{1}{2}} u_{1}\left(x_{1}, 0\right) \delta^{\prime}\left(x_{1}-\xi\right) d x_{1} \approx \int_{-\frac{1}{2}}^{\frac{1}{2}} \theta\left(x_{1}\right) \frac{\partial u_{0}}{\partial x_{1}}\left(x_{1}, a\right) \delta\left(x_{1}-\xi\right) d x_{1}
$$

which simplifies to

$$
-a \frac{\partial u_{1}}{\partial x_{1}}(\xi, 0) \approx \theta(\xi) \frac{\partial u_{0}}{\partial x_{1}}(\xi, a)
$$

By choosing the applied currents to be concentrated near the ends of the plate, e.g. by choosing

$$
\frac{\partial u_{0}}{\partial x_{3}}\left(x_{1}, 0\right)=-\phi\left(x_{1}\right)=-\delta\left(x_{1}+\frac{1}{2}-t\right)+\delta\left(x_{1}-\frac{1}{2}+t\right),
$$

for some $t>0$ small, we can make $\partial u_{0}\left(x_{1}, a\right) / \partial x_{1}$ nearly constant. Indeed, applying the thin plate approximation to $u_{0}$, we find that $u_{0}\left(x_{1}, x_{3}\right) \approx w\left(x_{1}\right)$, where

$$
\frac{d^{2}}{d x_{1}^{2}} w=-\frac{1}{a}\left[\delta\left(x_{1}+\frac{1}{2}-t\right)-\delta\left(x_{1}-\frac{1}{2}+t\right)\right], \quad \text { with } \quad \frac{d w}{d x_{1}}\left( \pm \frac{1}{2}\right)=0 .
$$


This gives

$$
\frac{\partial u_{0}}{\partial x_{1}}\left(x_{1}, x_{3}\right) \approx \frac{d w}{d x_{1}}\left(x_{1}\right)=-\frac{1}{a}, \quad\left|x_{1}\right| \leq \frac{1}{2}-t
$$

so that

$$
\frac{\partial u_{1}}{\partial x_{1}}(\xi, 0) \approx \frac{1}{a^{2}} \theta(\xi) \quad|\xi| \leq \frac{1}{2}-t .
$$

This formula shows that the data $u_{1}\left(x_{1}, 0\right)$ is related to the unknown loss profile $\theta\left(x_{1}\right)$ in a very simple manner. As a first approximation, with the proper input current, one can obtain the loss profile as a scaled derivative of the voltage difference between the damaged specimen and the undamaged specimen on the bottom side of the plate. We now develop an inversion procedure in 3-D (i.e. for thin plates) using similar arguments.

6. A method for reconstructing profile loss in 3-D. We begin by observing that the following identity

$$
\bar{\nabla} \cdot\left[\theta\left(\bar{\nabla} u_{0}\right) v_{0}\right]=\left(\bar{\nabla} \theta \cdot \bar{\nabla} u_{0}\right) v_{0}+\theta\left(\bar{\triangle} u_{0}\right) v_{0}+\theta\left(\bar{\nabla} u_{0} \cdot \bar{\nabla} v_{0}\right)
$$

holds for fixed $x_{3}$. Recall that $\bar{\nabla}$ denotes the two dimensional gradient $\left(\partial / \partial x_{1}, \partial / \partial x_{2}\right)$.

Using the thin plate approximation of Section 4, we find that

$$
u_{0}\left(x_{1}, x_{2}, x_{3}\right) \approx w\left(x_{1}, x_{2}\right) \text { where } \bar{\triangle} w=-\frac{1}{a} \phi\left(x_{1}, x_{2}\right),
$$

with the boundary condition $\partial w / \partial \nu=0$ on $\partial \omega$. Supposing a loss profile, $\theta$, with compact support, we select input current $\phi\left(x_{1}, x_{2}\right)$ with the property

$$
\{\operatorname{supp} \phi\} \bigcap\{\operatorname{supp} \theta\}=\emptyset .
$$

Practically, this means that our inspection method is designed to image an area where it is apriori suspected that some damage has occured by setting up current sources and sinks away from the damaged area. Therefore, the term $\theta\left(\bar{\triangle} u_{0}\right)$ is always zero (approximately).

Using this fact and substituting the identity (12) into (11), we get

$a \int_{\omega} u_{1}\left(x_{1}, x_{2}, 0\right) \zeta\left(x_{1}, x_{2}\right) d x_{1} d x_{2} \approx \int_{\omega} \bar{\nabla} \cdot\left[\theta\left(\left.\bar{\nabla} u_{0}\right|_{x_{3}=a}\right) v_{0}\right] d x_{1} d x_{2}-\int_{\omega}\left(\left.\bar{\nabla} \theta \cdot \bar{\nabla} u_{0}\right|_{x_{3}=a}\right) v_{0} d x_{1} d x_{2}$.

The first term on the right can be written as an integral over $\partial \omega$ by the divergence theorem. Since $\partial u_{0} / \partial \nu=0$ on $\partial \omega$, this integral is zero. We are therefore left with the approximate identity

$$
a \int_{\omega} u_{1}\left(x_{1}, x_{2}, 0\right) \zeta\left(x_{1}, x_{2}\right) d x_{1} d x_{2} \approx-\int_{\omega}\left(\left.\bar{\nabla} \theta \cdot \bar{\nabla} u_{0}\right|_{x_{3}=a}\right) v_{0} d x_{1} d x_{2} .
$$

Next, we narrow our choice of $\phi$ further. Observe from (13) that because of the square plate geometry, if we set $\phi$ to be independent of $x_{2}$, then we can make $\partial w / \partial x_{2}=0$. A natural choice is

$$
\phi\left(x_{1}, x_{2}\right)=\delta\left(x_{1}+\frac{1}{2}-t\right)-\delta\left(x_{1}-\frac{1}{2}+t\right)
$$

(see Figure 2). This current pattern generates, approximately, a constant current sheet directed in the $x_{1}$ direction. The desired background potential $u_{0}$ is computed using (13) leading to

$$
\frac{\partial u_{0}}{\partial x_{1}}\left(\bar{x}, x_{3}\right) \approx \frac{d w}{d x_{1}}\left(x_{1}\right)=-\frac{1}{a}, \quad\left|x_{1}\right|<\frac{1}{2}-t .
$$




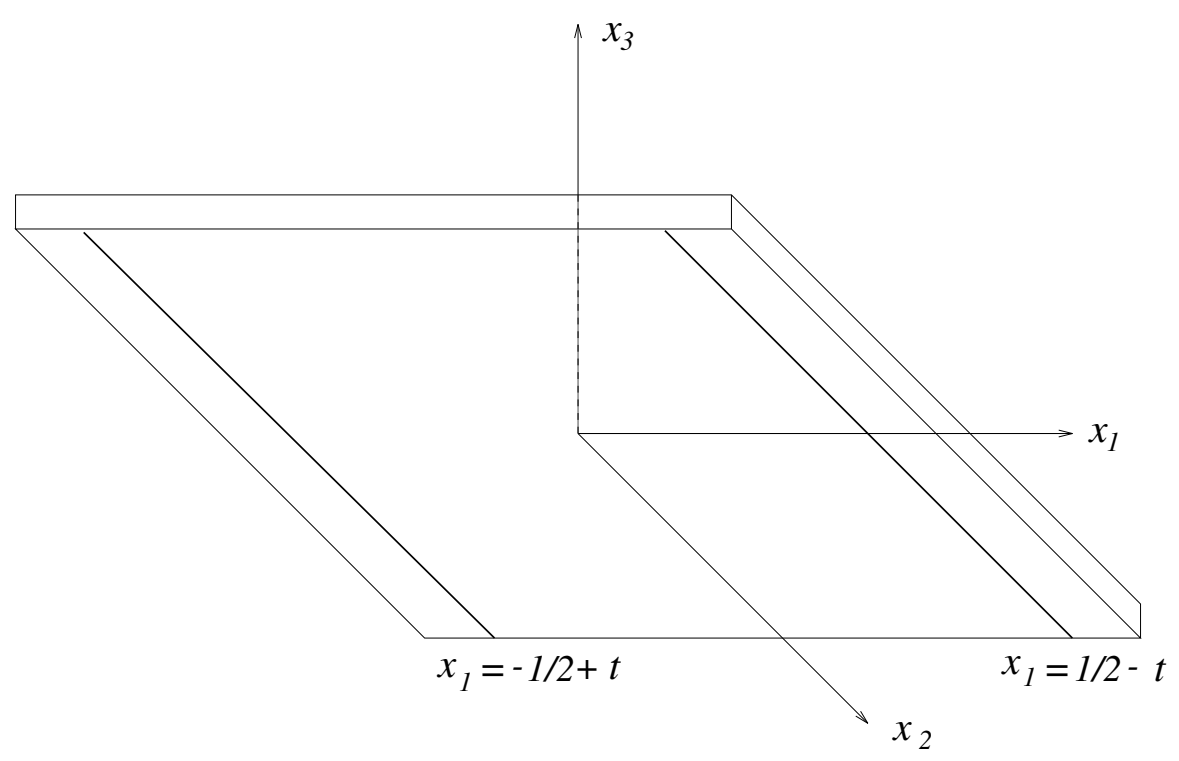
shown).

FIG. 2. Current is applied along $\left|x_{1}\right|=\frac{1}{2}-t$. The damage to be imaged is on the top side of the plate (not

With this choice of current pattern, (14) reduces to

$$
a \int_{\omega} u_{1}\left(x_{1}, x_{2}, 0\right) \zeta\left(x_{1}, x_{2}\right) d x_{1} d x_{2} \approx \frac{1}{a} \int_{\omega} \frac{\partial \theta}{\partial x_{1}} v_{0} d x_{1} d x_{2}
$$

Our method is to set, formally, $v_{0}=\delta\left(x_{1}-\xi_{1}\right) \delta\left(x_{2}-\xi_{2}\right)$, corresponding to $\zeta=\bar{\triangle}\left[\delta\left(x_{1}-\xi_{1}\right) \delta\left(x_{2}-\xi_{2}\right)\right]$. We thus obtain

$$
\bar{\triangle} u_{1}\left(\xi_{1}, \xi_{2}, 0\right) \approx \frac{1}{a^{2}} \frac{\partial \theta}{\partial x_{1}}\left(\xi_{1}, \xi_{2}\right) .
$$

This last formula may be viewed as a direct, approximate reconstruction scheme. Data collected are represented by $u_{1}\left(\xi_{1}, \xi_{2}, 0\right)$. We can view the equation as a first order differential equation for $\theta$. Null values for $\theta$ on an appropriately chosen curve permit us to integrate the equation.

6.1. A direct marching algorithm. Suppose we have measured $u_{1}\left(x_{1}, x_{2}, 0\right)$ at a discrete set of points

$$
\left(x_{1}^{(i)}, x_{2}^{(j)}\right), \quad \text { for } \quad i=0,1, \cdots, m, j=0,1, \cdots, n .
$$

We assume that the points are equidistant with $\left(x_{1}^{(i)}-x_{1}^{(i-1)}\right)=\left(x_{2}^{(j)}-x_{2}^{(j-1)}\right)=h$. Let us denote the data by

$$
u_{1}\left(x_{1}^{(i)}, x_{2}^{(j)}, 0\right)=g(i, j) .
$$

We approximate the Laplacian of $u_{1}\left(x_{1}, x_{2}, 0\right)$ at $\left(x_{1}^{(i)}, x_{2}^{(j)}\right)$ by the five point stencil

$$
f(i, j):=\frac{1}{h^{2}}[g(i+1, j)+g(i-1, j)+g(i, j+1)+g(i, j-1)-4 g(i, j)] .
$$

We also discretize the unknown $\theta\left(x_{1}, x_{2}\right)$ and approximate its partial derivative with respect to $x_{1}$ by a forward difference. As a result (17) is approximated discretely by

$$
f(i, j)=\frac{1}{a^{2} h}\left(\theta\left(x_{1}^{(i+1)}, x_{2}^{(j)}\right)-\theta\left(x_{1}^{(i)}, x_{2}^{(j)}\right)\right) .
$$


Let us assume that we have the boundary conditions $\theta\left(x_{1}^{(0)}, x_{2}^{(j)}\right)=0$. Then we can use (18) to compute $\theta\left(x_{1}^{(i)}, x_{2}^{(j)}\right)$ for $i>0$.

6.2. A regularized method. As we will demonstrate with numerical examples, the direct marching scheme, despite its simplicity, does not in general perform well. As a remedy we propose a regularization of the inverse problem that is quite effective in removing some of the undesirable characteristics of the direct marching algorithm.

The main reason why the direct marching algorithm does not perform well is that the data $g(i, j)$, and hence $f(i, j)$, only approximately correspond to a loss profile function $\theta$ that has compact support. We recall that this was an essential assumption for the development of our method. If it is not exactly satisfied for the data, there may result some quite disturbing shadow effects. Ideally, we know that

$$
\operatorname{supp} \theta \subset \subset \omega_{0}
$$

If the data $g(i, j)$ are inconsistent, then it makes sense to project $g(i, j)$ so that it is in the range of $\theta$ which are supported in $\omega_{0}$. Such a procedure, however, also introduces unwanted artifacts.

A second reason that the reconstruction from direct marching is often poor is that by the choice of input electrodes, and hence the background field $u_{0}$, we have decoupled $\theta\left(x_{1}^{(i)}, x_{2}^{(j)}\right)$ from its neighbor $\theta\left(x_{1}^{(i)}, x_{2}^{(j+1)}\right)$. Therefore, depending on the quality of the data $g(i, j)$, we can lose smoothness in the reconstructed $\theta$ along the $x_{2}$ axis. These points will be illustrated clearly in the numerical examples.

In order to overcome these difficulties, we propose to regularize the reconstruction with a smoothing operator. If we are given $u_{1}\left(x_{1}, x_{2}, 0\right)=g\left(x_{1}, x_{2}\right)$ as data, we suggest solving

$$
\begin{aligned}
& \min \left\{\left\|a^{2} \bar{\triangle} g-\frac{\partial \theta}{\partial x_{1}}\right\|^{2}+\lambda\|\bar{\nabla} \theta\|^{2}\right\} \\
& \text { subject to } \theta=0 \text { on } \partial \omega_{0} .
\end{aligned}
$$

The term involving $\bar{\nabla} \theta$ has the effect of smoothing the reconstructed $\theta$. This smoothing is achieved at the cost of a loss of fit to the data.

The procedure above can be given a partial differential equation description. The minimizer of (19) solves the boundary value problem

$$
\begin{gathered}
(1+\lambda) \frac{\partial^{2} \theta}{\partial x_{1}^{2}}+\lambda \frac{\partial^{2} \theta}{\partial x_{2}^{2}}=a^{2} \frac{\partial}{\partial x_{1}} \bar{\triangle} g \\
\theta=0 \text { on } \partial \omega_{0} .
\end{gathered}
$$

We propose to use the $L$-curve method [6] to find the parameter $\lambda$. It is worth noting that by adding the regularization, the reconstruction algorithm has become less direct. In effect, we need to solve an elliptic boundary value problem, which, after discretization, translates to a sparse matrix inversion.

7. Numerical experiments. We report some results from numerical calculations using the algorithm described in Section 6.2. In all the computations, the plate thickness $a$ is 0.10 (aspect ratio of 1 -to-10).

Data for inversion are generated using the boundary element method and represent the full three dimensional, nonlinear relationship between the loss profile and measured voltage. Instead of the Neumann problem (1), we alter the problem slightly and replace the boundary conditions 

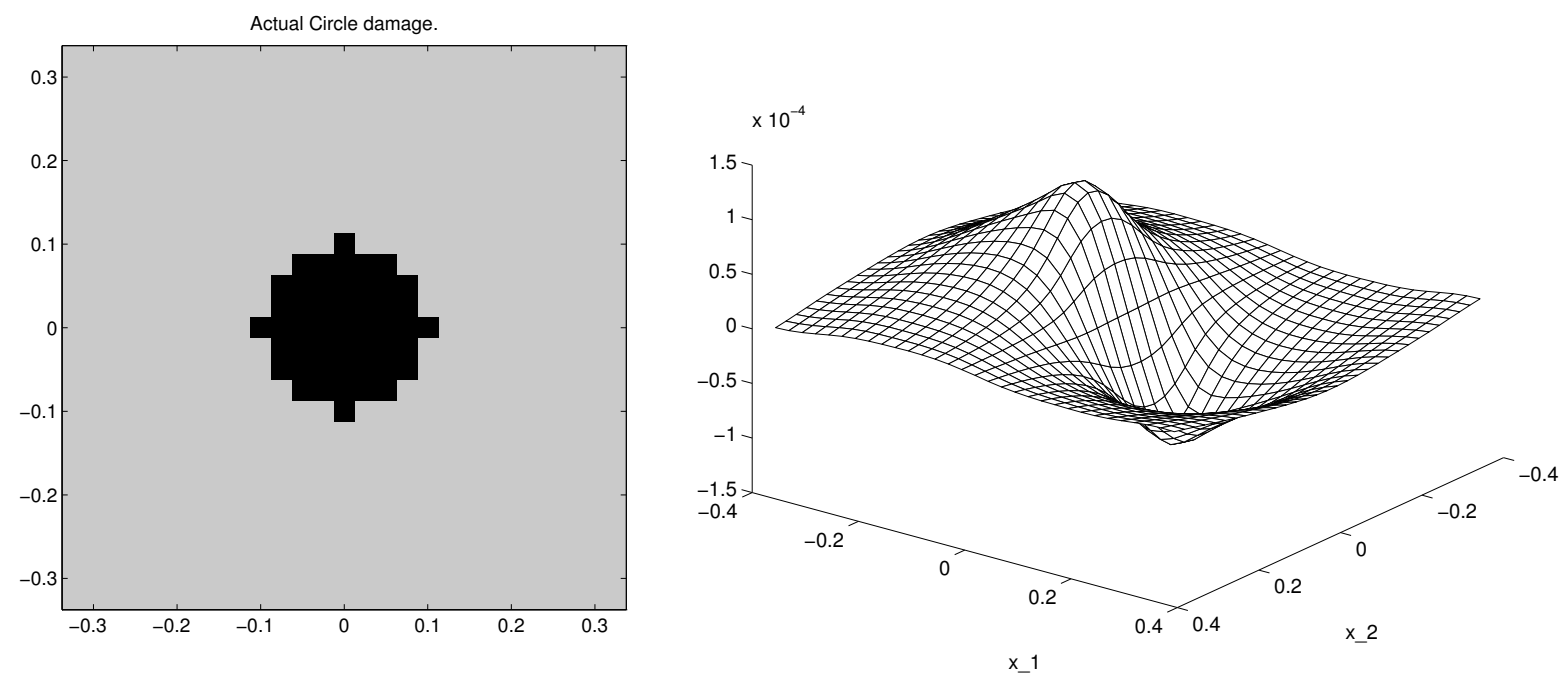

FIG. 3. Image of the circular damage and the corresponding boundary voltage $u_{\epsilon}\left(x_{1}, x_{2}, 0\right)-u_{0}\left(x_{1}, x_{2}, 0\right)$.

at $x_{1}= \pm 1 / 2$ with homogeneous Dirichlet conditions. This was done in order to allow the use of a conjugate residual algorithm [3] to solve the resulting square linear system coming from the boundary element method. Recall that the original boundary value problem was unique up to a constant. Using the normalization described in Section 2, we would get a non-square linear system, which would have to be appropriately transformed before application of a conjugate residual method. Instead of delta function input currents, we use an approximation using piecewise linear functions. We emphasize that the change in the boundary condition and the approximation in the input current patterns do not affect the inversion algorithm in an essential way.

Current is distributed along strips parallel to the $x_{2}$-axis on the bottom of the plate. The strips are of width 0.1 and centered at $x_{1}= \pm 0.425$. The distribution is independent of $x_{2}$ in the form of a symmetric triangle reaching the maximum height of 1 . We absorb the parameter $\epsilon$ into $\theta$ and specify the loss profile $\theta\left(x_{1}, x_{2}\right)$ at the nodes of a uniform mesh of size $1 / 40$. Values of $\theta$ at all other points are obtained using bilinear interpolation.

The data needed for inversion is computed by first selecting a loss profile $\theta\left(x_{1}, x_{2}\right)$ and solving for the corresponding boundary values $u\left(x_{1}, x_{2}, 0\right)$. Then we compute the corresponding boundary values for the undamaged plate. The difference in the boundary voltages between the damaged plate and the undamaged plate represents our data. Note that the data computed this way are truly three dimensional and depend nonlinearly on the loss profile, whereas our reconstruction method is based on linearization and a two dimensional approximation. Data are presumed known on a 27 -by-27 square array of points over the region $[-0.325,0.325] \times[-0.325,0.325]$.

In Figure 3, we display a "circular" damage and its corresponding data. The image of the damage is misleading since it is displayed over a coarse array of pixels of size $1 / 40$. The pixel value is the value of $\theta$ at the node centered on the pixel without bilinear interpolation. The depth of the profile is 0.005 ( $5 \%$ thickness loss).

The data obtained are used in the inversion with $\lambda=0$ and $\lambda=1 / 8$. The results of the reconstruction are shown in Figure 4. Note that increasing $\lambda$ has the effect of smoothing the recontruction.

The $L$-curve is a plot of the data fit against the amount of regularization for different values of $\lambda$. The principle behind the $L$-curve is that the value of the regularization parameter is optimal at the 'elbow' of the curve because at that point we have balanced the data fit with the amount 

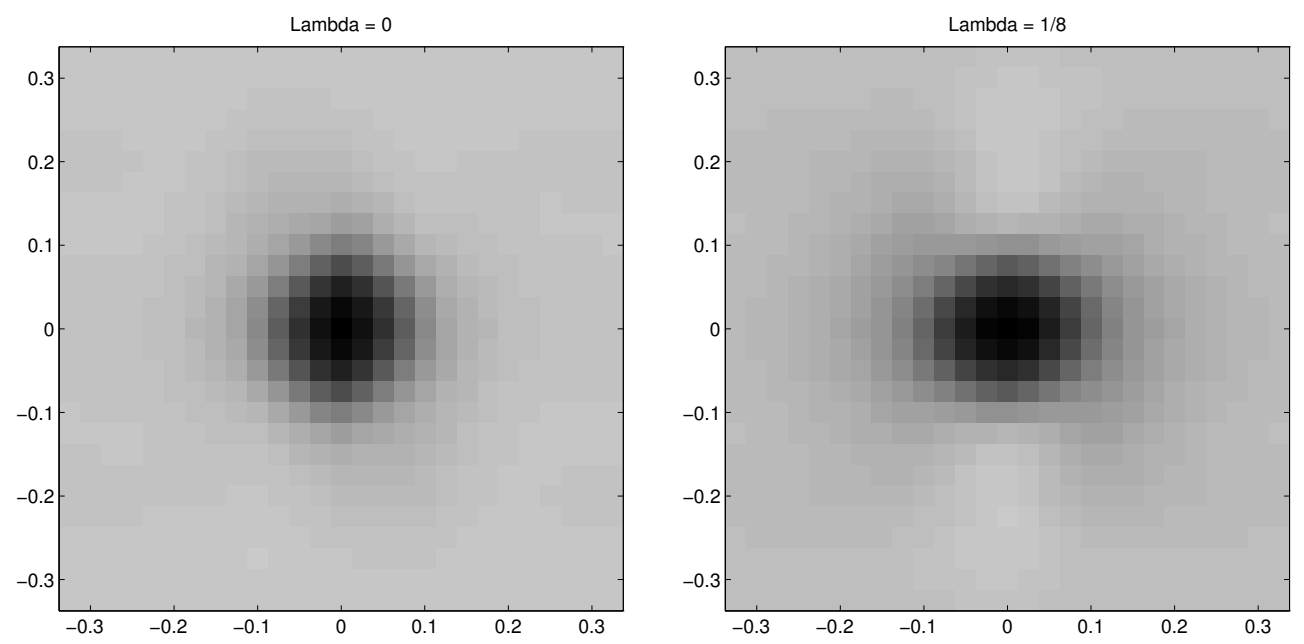

FIG. 4. Images of the reconstruction of the circular damage for $\lambda=0$ (left) and $\lambda=1 / 8$ (right). Note the smoothing effect of $\lambda$. The accompanying L-curve is shown in Figure 5.

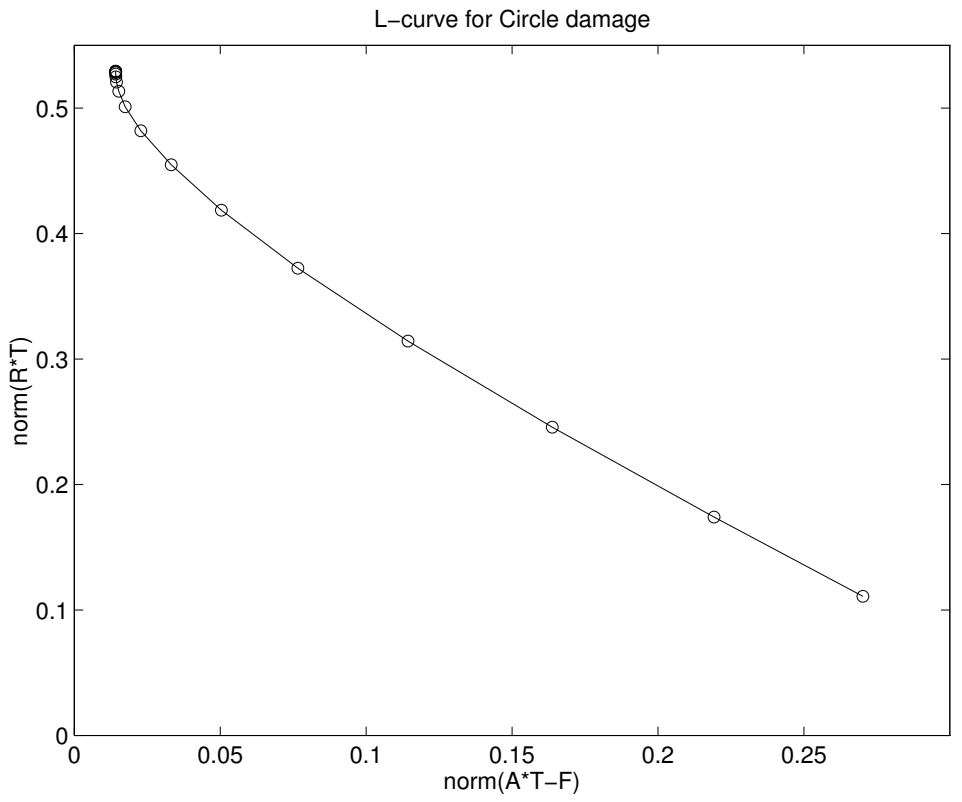

FIG. 5. The L-curve associated with the circle damage. The elbow of the curve appears to be near the left end of the curve, $\lambda=0$. 
Reconstructed Circle damage: lambda $=0$

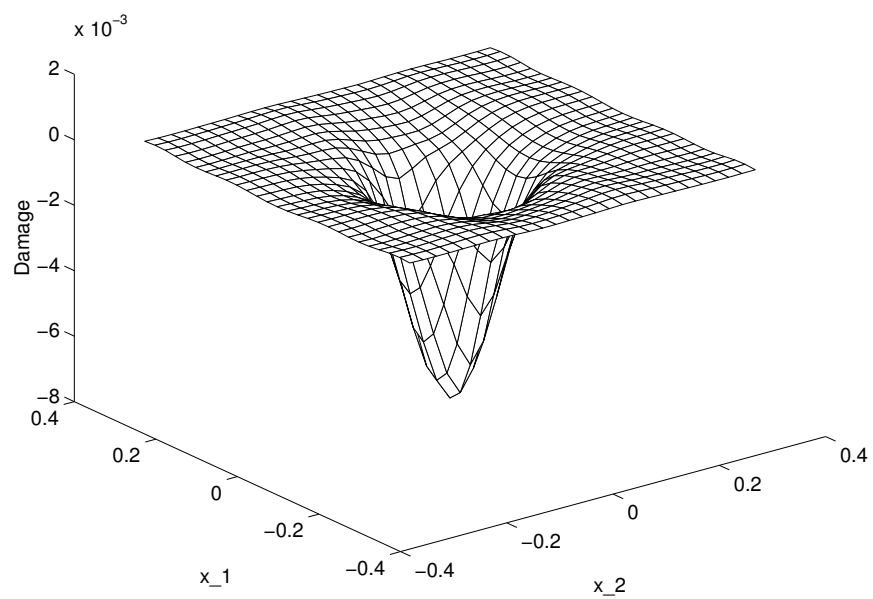

FIG. 6. Plot of the reconstructed circular damage. Note that the thickness loss, which is 0.005 is accurately estimated.

regularization. For this example, the $L$-curve (see Figure 5 ) does not have a pronounced elbow. Inspection of the curve reveals that the elbow is near $\lambda=0$, the left end of the curve. We do not have an algorithm that picks the optimal value of $\lambda$ automatically. Instead, we rely on graphing the entire curve, which is quite inexpensive due to the sparsity of the linear system, and choosing a value for $\lambda$.

A more informative display is the 3 -D plot of the reconstructed loss profile for $\lambda=1 / 8$, displayed in Figure 6. Note that the true depth, which is 0.005 , is quite accurately estimated by our reconstruction.

For the next example, we choose a square damage which is off-center relative to the measurement array. The depth of the profile is 0.01 ( $10 \%$ thickness loss). Images of the true profile and its reconstruction with $\lambda=1 / 16$ are displayed in Figure 7 . The value $\lambda$ is arrived at by inspecting the $L$-curve, which is shown in Figure 8. For this example, the $L$-curve does have a recognizable elbow, which appears near $\lambda=1 / 16$.

The surface plot of the reconstruction is given in Figure 9. Note that the true depth of the loss profile, which is 0.01 ( $10 \%$ material loss), is well estimated by the reconstruction. Observe the shadow artifacts and the overshoot in the recovered profile.

When we pick smaller values of $\lambda$, the shadow artifacts are more pronounced. We display an image of the reconstruction for $\lambda=0$. This value of $\lambda$ corresponds to the unregularized case, i.e., the direct marching scheme outlined in Section 6.1, and can be seen to produce poor reconstruction. We were unable to remove the striping artifacts by preprocessing the data so that they are consistent with the linearized inverse problem for loss profiles that are compactly supported. We found that an effective way to minimize this artifact and to control the smoothness of the reconstruction is to add the regularization mentioned earlier.

We ran several more examples with similar findings. Experiments in which small amounts of noise were added to the data were also conducted. The use of regularization allowed reconstruction of comparable quality.

8. Discussion. We have developed a method for obtaining quantitative information about corrosion damage from measurements of voltages and currents on the boundary. The computational algorithm that is used to invert the data is based on linearization and a thin plate approximation. 

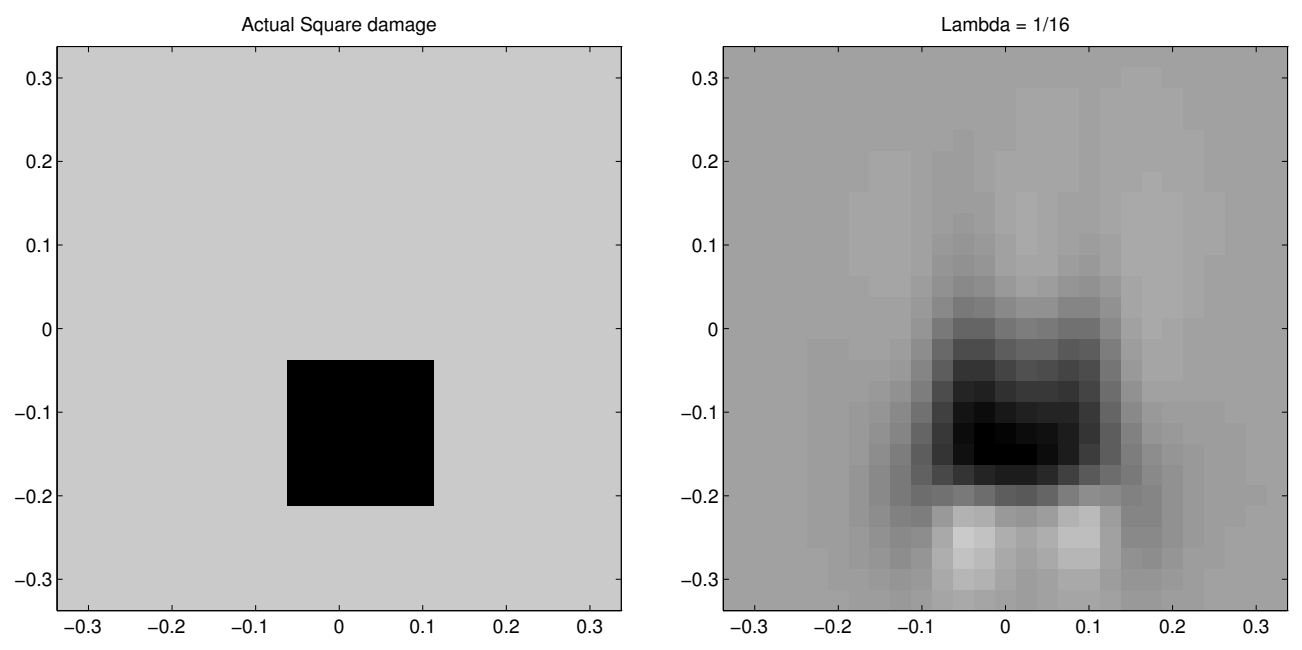

FIG. 7. Images of the true square damage and its reconstruction for $\lambda=1 / 16$.

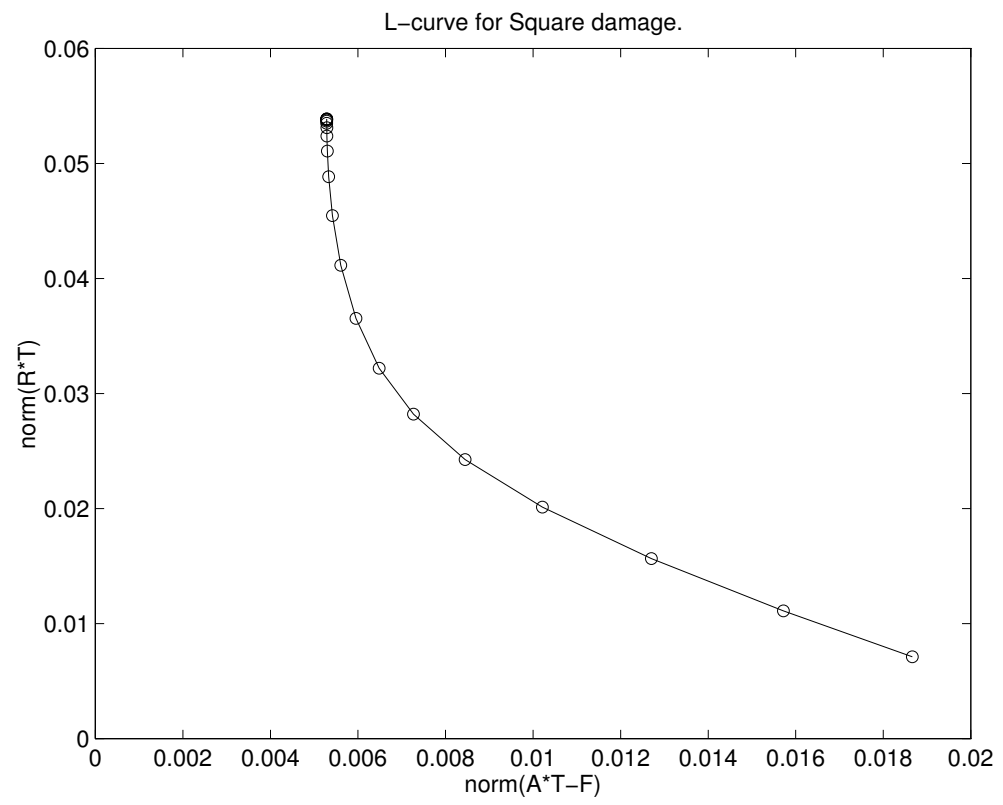

FIG. 8. The L-curve associated with the square damage. The elbow of the curve is near $\lambda=1 / 16$. 
Square damage reconstructed: lambda $=1 / 16$

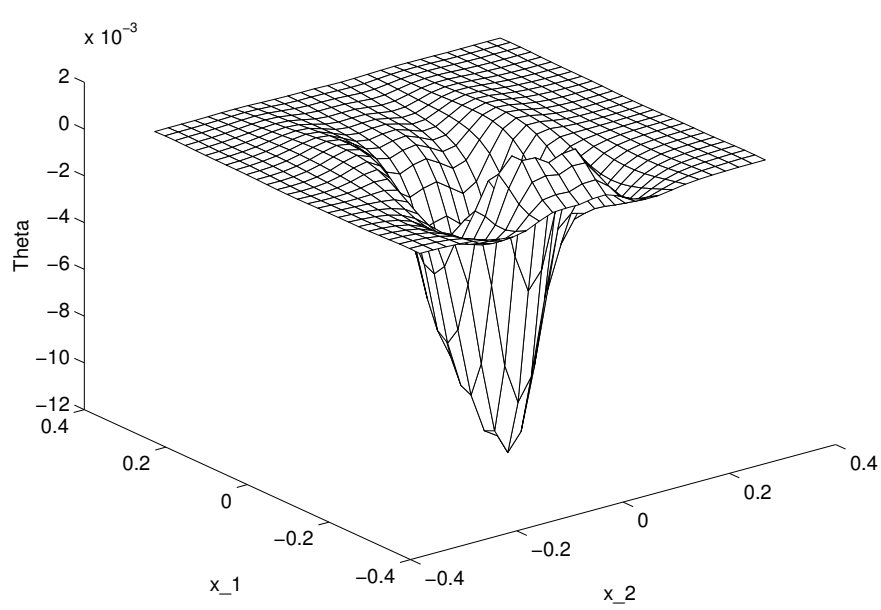

FIG. 9. Plot of the reconstructed square damage. Note that the thickness loss, which is 0.01 is accurately estimated.

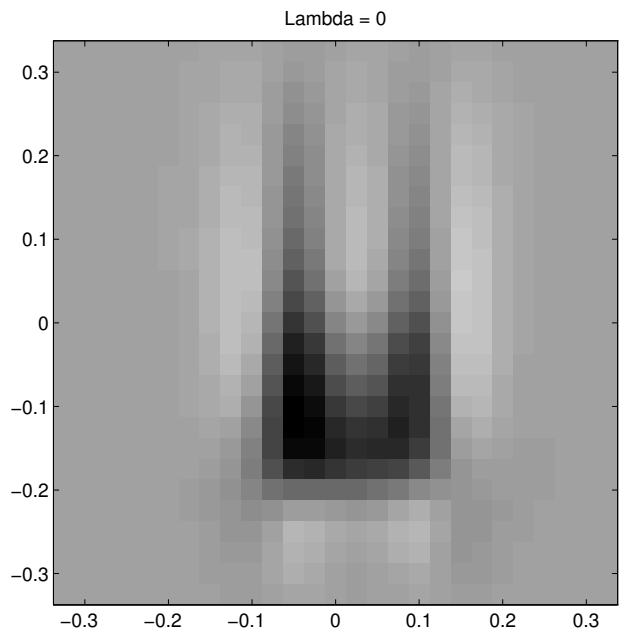

FIG. 10. Reconstruction for $\lambda=0$ (unregularized). Note that the shadow artifacts in the image are removed by regularization. 
The accuracy of the method is demonstrated in numerical examples.

The reconstruction algorithm is quite effective. While we did not implement a procedure that automatically determines the optimal regularization parameter, we believe that this can be done. The question of obtaining data of the quality required is more difficult to answer. Preliminary results from a laboratory experiment conducted by Ian Hall [4] indicated that the high accuracy data needed for our method posed certain difficulties. However, more recent indications are that it may be possible to obtain data of sufficiently high quality in the laboratory setting. On the other hand, the ability of the method to detect small material loss, of the order of 5 to $10 \%$, suggests that research in instrumentation for this method may be worthwhile.

Acknowledgements. This paper was written during Peter Kaup's and Fadil Santosa's visit to the Advanced Computing Research Institute, Cornell Theory Center. They thank Tom Coleman, the Instutute's director, for the hospitality and support.

\section{REFERENCES}

[1] ASM Metals Handbook, Ninth Edition, Vol. 13, Corrosion, ASM International, Metals Park, OH, 1987.

[2] S. Andrieux, A. Ben AbDA, AND M. JAOuA, Identifiabilité de frontière inaccessible par des mesures de surface, C. R. Acad. Sci. Paris, 316-1 (1993), pp. 429-434.

[3] S. Eisenstat, H. Elman, And M. Schultz, Variational iterative methods for nonsymmetric systems of linear equations, SIAM J. Numer. Anal., Vol. 20 (1983), pp. 345-357.

[4] I. HALL, personal communication, 1994-95.

[5] R. Halmshaw, Non-destructive Testing, Wiley-Interscience, 1987.

[6] P. Hansen, Analysis of discrete ill-posed problems by means of the L-curve, SIAM Review, Vol. 34 (1992), pp. $561-580$.

[7] P. Kaup And F. Santosa, Nondestructive evaluation of corrosion damage using electrostatic boundary measurements, to appear in J. Nondestructive Evaluation.

[8] M. Vogelius And J. XU, A nonlinear elliptic boundary value problem related to corrosion modeling, in progress. 